EUROPEAN ORGANIZATION FOR NUCLEAR RESEARCH

CERN - AB DEPARTMENT

CERN-AB-2006-085

CLIC Note 700

\title{
Observations about RF Breakdown from the CLIC High- Gradient Testing Program
}

W. Wuensch

\begin{abstract}
One of the most important objectives of the CLIC (Compact Linear Collider) study is to demonstrate the design accelerating gradient, which in the current parameter set targets $150 \mathrm{MV} / \mathrm{m}$, under practical operating conditions. A testing program has been put into place to achieve this objective. Recent advances in understanding and quantifying the effects which both limit the ultimate accelerating gradient and fix the practical operating gradient are presented.
\end{abstract}

Keywords: linear collider, accelerating structure, high gradient, rf, breakdown PACS: 07.57.-c 


\section{INTRODUCTION}

Although metal accelerating structures have been shown to be capable of sustaining an accelerating gradient above $200 \mathrm{MV} / \mathrm{m}$ [2] the constraints imposed by a linear collider application result in a practical accelerating gradient that is much lower. As a consequence, a large part of the effort to maximize the accelerating gradient for a linear collider must include understanding and quantifying the effects which limit gradient.

One of the central design compromises in a linear collider is between accelerating gradient and the luminosity to power-consumption ratio, which is the basic figure of merit for the energy efficiency [3]. This compromise can be observed in individual parameters. A short rf pulse is desirable for both $\mathrm{rf}$ breakdown and for pulsed surface heating, however is less efficient because the bunch-train length to rf fill time ratio is lower. A small aperture structure, low $\mathrm{a} / \lambda$, is desirable for having low surface electric field and power flow to accelerating-gradient ratios however has higher short range wakefields which lead to emittance growth and luminosity reduction. Higher-ordermode features reduce emittance growth increasing efficiency but concentrate surface electric and magnetic fields and local power flow thus lowering high-gradient performance.

Quantifying all of these the effects becomes crucial for maximizing a linear collider gradient. With an accurate function predicting the gradient that a structure will achieve, a wide range of structures and rf pulse parameters can be considered and the compromise between gradient and efficiency can be understood and optimized [3]. The next section in this report describes recent advances in understanding one of these limits, maximum gradient given by power flow through a structure. In the final section, the breakdown probability of individual accelerating structures which limits the practical accelerating gradient to a value below the maximum to which the structure can be conditioned, is discussed. Recent measurements of breakdown probability as a function of a number of parameters including gradient and material as well as a proposed explanation for the breakdown probability and the slope with gradient are presented.

\section{POWER LIMIT SCALING}

The accelerating gradient limit for traveling-wave accelerating structures has been observed to depend on surface electric field, and lowering the peak surface electric field to accelerating gradient ratio has been used to improve the performance of structures [4]. There is clear evidence however, in data from both CLIC and NLC [5,6] which covers structures with a wide range of rf parameters, that a simple constant surface field limit is insufficient to predict the performances of different structures. In general the data shows that larger aperture and higher group velocity structures tolerate lower surface electric fields. They do on the other hand support much higher power flows. An attempt to explain this and to quantify the limit has led to the suggestion that traveling wave structures have a material dependent limit which is given by, 


$$
\frac{P \tau^{\alpha}}{C}<\text { Const }
$$

where $P$ is the power flow, $\tau$ is the pulse length, $\alpha$ gives the pulse length dependence (observed to be about $1 / 3$ for copper and $2 / 3$ for molybdenum) and $C$ is the smallest circumference of the structure [5]. A refined physical explanation for this power limit scaling and expanded data is presented in this report. The subsequent arguments will be made for constant pulse length and the pulse length dependence will be addressed again below.

The proposed limit is an extension of the ablation limit of $P \tau^{1 / 2}$ described in [7]. The physical reasoning for a power over circumference limit begins with the observation that the melted spots observed on structures after rf conditioning have a typical diameter of $100 \mu \mathrm{m}$ (see for example [8]), independent of frequency and structure parameters, and are thus small with respect to the features of structures in the frequency range of $30 \mathrm{GHz}$ and below. The power available to feed a discharge which produces such a small but fixed width spot is proportional to the power density, which is given by the total power flow through the structure divided by the circumference of structure.

During the conditioning process, the cumulative effect of breakdowns is to improve the field holding capacity of the structure surface. Above a certain threshold in discharge power however, the detrimental effect on surface geometry from a breakdown, surface roughening or even erosion and eventually 'damage', is greater than the improvement in field holding capacity. Conditioning saturates under these conditions and the power limit of the structure is reached. This corresponds to the limiting regime described in [9]. What is added by this proposed scaling is that the power available to the discharge is related to the geometry of the structure through the quantity $P / C$.

The pulse length dependence of the originally proposed $P \tau^{1 / 2}$ scaling is given by one dimensional heat diffusion during the breakdown. Observed pulse length dependencies, generally $\alpha \approx 1 / 3$ for copper [10] and $\alpha \approx 2 / 3$ [11] for molybdenum, differ somewhat. The lower value for copper may indicate that the heat diffusion is in more than one dimension or that another cooling process, such as radiation, is occurring. The higher value for molybdenum may be because of its lower thermal conductivity.

The validity of the scaling has been tested by considering data from a number of data sets for structures tested under comparable conditions. The data from a series of disk loaded waveguide structures are presented in table 1 . The value of $P \tau^{1 / 3} / C$ for the three $30 \mathrm{GHz}$ structures is remarkably consistent even though the values of group velocity differ by an order of magnitude, the surface electric field differs by over a factor of two and the total power flow differs by a factor of three in the opposite direction. The values of $P \tau^{1 / 3} / C$ for the two X-band structures are also very similar for very different types of structures, although they are somewhat higher than at $30 \mathrm{GHz}$. This could be either due to a residual frequency dependence or to another structure dimension such as iris thickness. The irises of the $30 \mathrm{GHz}$ structures are $0.85 \mathrm{~mm}$ which may give more limited heat conduction during breakdown for the estimated $0.1 \mathrm{~mm}$ diameter discharges. 
TABLE 1. $30 \mathrm{GHz}$ copper periodic structure data $[12,13,14,15,16]$. The structure labeled NLC is structure number H60VG4S17 and data is quoted for the first cell. $2 a$ refers to iris diameter.

\begin{tabular}{ccccccccc}
\hline & $\begin{array}{c}\boldsymbol{f} \\
{[\mathbf{G H z}]}\end{array}$ & $\boldsymbol{V}_{\mathbf{g}} / \mathbf{c}$ & $\begin{array}{c}\boldsymbol{E}_{\text {acc }} \\
{[\mathbf{M e V} / \mathbf{m}]}\end{array}$ & $\begin{array}{c}\boldsymbol{E}_{\text {surf }} \\
{[\mathbf{M e V} / \mathbf{m}]}\end{array}$ & $\begin{array}{c}\boldsymbol{P} \\
{[\mathbf{M W}]}\end{array}$ & $\begin{array}{c}\boldsymbol{\tau} \\
{[\mathbf{n s}]}\end{array}$ & $\begin{array}{c}\mathbf{2 a} \\
{[\mathbf{m m}]}\end{array}$ & $\frac{P \tau^{1 / 3}}{C}$ \\
\hline $\begin{array}{c}\text { CERN } \\
\text { X-band }\end{array}$ & 11.424 & 0.011 & 153 & 326 & 69 & 150 & 6 & 19 \\
$\begin{array}{c}\text { NLC } \\
\text { Acceler- }\end{array}$ & 11.424 & 0.045 & 72 & 152 & 140 & 100 & 11.4 & 18 \\
$\begin{array}{c}\text { ating } \\
\text { CTF2 }\end{array}$ & 30 & 0.047 & 116 & 253 & 34 & 70 & 3.5 & 13 \\
$\begin{array}{c}\text { PETS } \\
\text { CTF3 }\end{array}$ & 30 & 0.5 & & & 240 & 16 & 16 & 12 \\
PETS & 30 & 0.40 & 30 & 116 & 100 & 50 & 9 & 13 \\
\hline
\end{tabular}

The validity of the $P \tau^{1 / 3} / C$ limit applied to rectangular waveguide has been investigated using an experiment made at SLAC [17]. In the experiment two different width copper waveguides were investigated and data taken from the reference is presented in table 2. The circumference taken in the $\mathrm{TM}_{0,1}$ structures has been replaced by twice the width of $\mathrm{TE}_{1,1}$ mode of the rectangular waveguide, the part of the waveguide circumference with normal electric field.

The relative consistency of the power over circumference limit for the waveguides is again remarkable despite the large differences between the other parameters. The waveguide limit is somewhat lower than the X-band circular limit. This could be because the power flow is not constant across the width of the waveguide crosssection so the local power flow may is somewhat higher. A more precise comparison would be to compare the maximum Poynting vector integrated over an appropriate cross section.

TABLE 2. Copper waveguide data taken from [20]. $a$ is the waveguide width.

\begin{tabular}{cccccccc}
\hline & $\begin{array}{c}\mathbf{f} \\
{[\mathbf{G H z}]}\end{array}$ & $\boldsymbol{V}_{\mathbf{g}} / \mathbf{c}$ & $\begin{array}{c}\boldsymbol{E}_{\text {surf }} \\
{[\mathbf{M e V} / \mathbf{m}]}\end{array}$ & $\begin{array}{c}\boldsymbol{P} \\
{[\mathbf{M W}]}\end{array}$ & $\begin{array}{c}\boldsymbol{\tau} \\
{[\mathbf{n s}]}\end{array}$ & $\begin{array}{c}\boldsymbol{a} \\
{[\mathbf{m m}]}\end{array}$ & $\frac{P \tau^{1 / 3}}{2 a}$ \\
\hline $\begin{array}{c}\text { WR-90 } \\
\text { Reduced } \\
\text { width }\end{array}$ & 11.424 & 0.82 & 60 & 56 & 750 & 22.9 & 11.2 \\
\hline
\end{tabular}

\section{BREAKDOWN PROBABLILITY}

One of the key issues for reliable high luminosity operation of a linear collider is the breakdown probability of the individual accelerating structures [18]. Breakdowns in accelerating structures will randomly cause loss in acceleration and, potentially more seriously, transverse kicks to the beam. Such transverse kicks have been measured to be about $10 \mathrm{KeV}$ in X-band accelerating structures in the NLCTA [6]. A simple order of magnitude estimate for an acceptable breakdown probability for CLIC, based on the assumptions that all luminosity is lost on a pulse with a breakdown and that not more than $10 \%$ of total luminosity should be lost, gives that the breakdown probability for a structure should be $10^{-6}$ since there will be approximately $10^{5}$ accelerating structures in CLIC. This is a very low probability which has serious consequences on a practical operating gradient of a linear collider. 
Breakdown probability data from tests of four structures is shown in fig. 1. The data are from an NLC structure [10] and a series of tests of identical geometry CLIC structures: a $30 \mathrm{GHz}$ molybdenum iris structure [11], a $30 \mathrm{GHz}$ copper structure [12] and an X-band molybdenum iris structure [8]. The data have been taken at different pulse lengths and also conditioning state in the case of the $30 \mathrm{GHz}$ copper data. In all the three structures the breakdown probability at a given gradient could only be improved by conditioning the structure. No long term improvement in breakdown rate was observed during the low breakdown probability operation which lasted for about one hundred hours.

The breakdown probability of a structure appears in all cases to fall off exponentially as the gradient is reduced. The data are fit with two groups of lines on the linear/log plot in Fig. 1, one group for copper and another group for molybdenum. Each individual line is fit with a single parameter $E\left(10^{-1}\right)$, the gradient giving $10^{-1}$ breakdown probability. The slope of each fit line, in units of decades of probability per $\mathrm{MeV}$, is given by $1 / 0.09 E\left(10^{-1}\right)$ for molybdenum data and $1 / 0.06 E\left(10^{-1}\right)$ for copper data.

That the data can be fit in such a way gives insight into two major effects. One is that there appears to be a dependence of the slope on material. The other is that the breakdown probability, for a given pulse length, is determined entirely by the highest gradient at which the structure can be conditioned to, independent of frequency, structure geometry and conditioning state.

There are a number of consequences of these effects. The first is that while a material like molybdenum has a clearly higher peak gradient capability compared to copper, the lower breakdown probability slope means that at a certain probability it will be no better. Intriguingly the cross over point for copper and molybdenum appears to be at around $10^{-6}$, which corresponds to the estimated requirement for CLIC. This implies that the dominant effect considered in material studies for linear colliders should be breakdown rate. On the other hand, other applications which do not require such a low breakdown probability may be able to profit from molybdenum. Another effect is that the breakdown probability is determined by the ultimate gradient, the value of which is explained by the arguments of and referred to in the previous section. The inverse proportionality of the slopes to gradient at fixed probability also implies that the pulse length dependence is constant for all breakdown probabilities - probability lines of different pulse length converge at lower gradient so as to maintain the same pulse length dependence. 


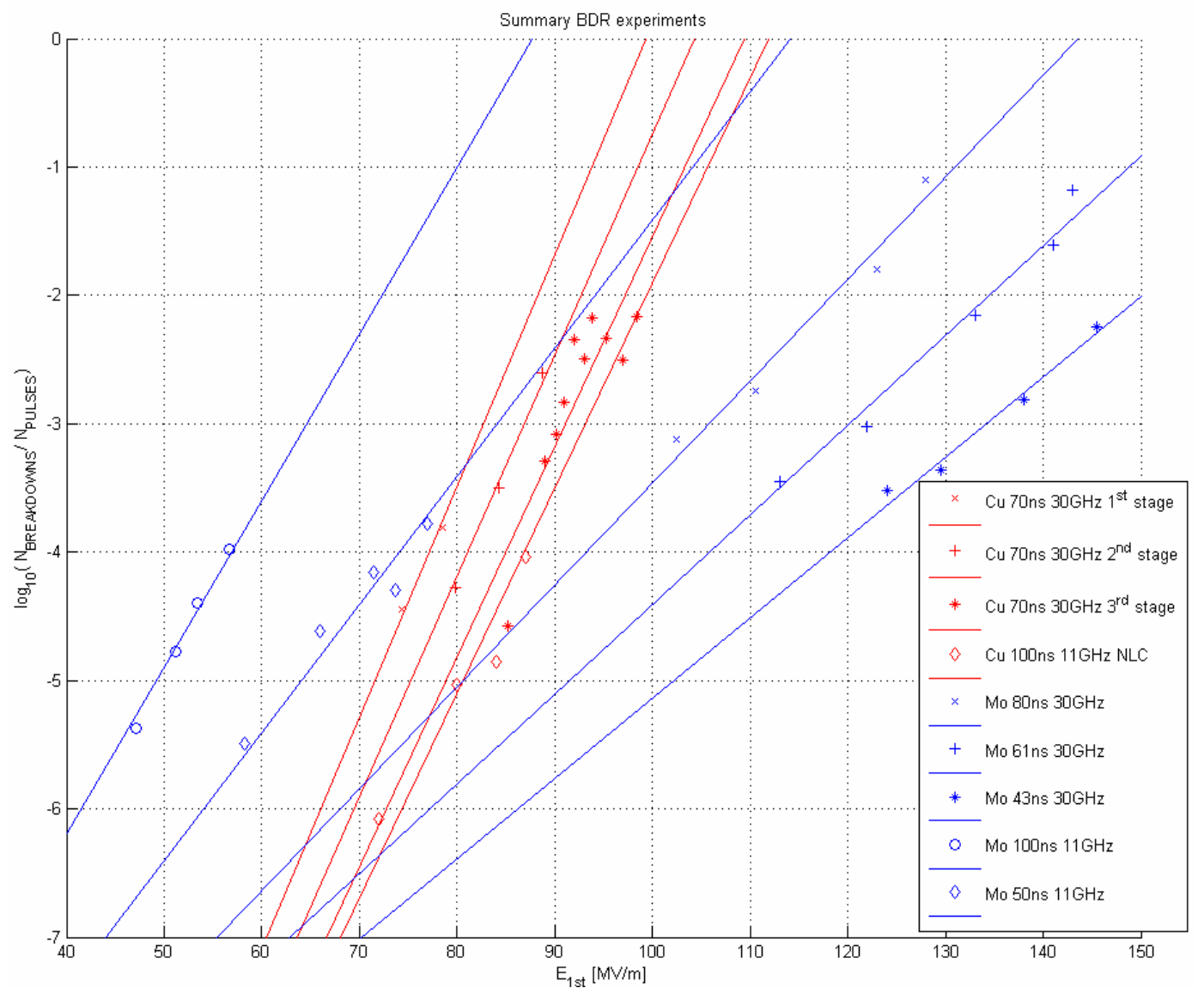

FIGURE 1. Breakdown rate measurements. The copper data is plotted in red and fit with $1 / 0.06 E\left(10^{-1}\right)$ and the molybdenum is plotted in blue and fit with $1 / 0.09 E\left(10^{-1}\right)$. The three $30 \mathrm{GHz}$ copper data sets were taken at successive moments in the conditioning process, hence the improving breakdown probability at fixed gradient.

Although the data provides very compelling evidence for the arguments which have been presented, the understanding emerged after the data was taken. As a consequence further tests are required to verify their validity and to address possible errors. One of the main questions is the effect clamped irises (rather than brazed) have on the molybdenum data, since both the $11 \mathrm{GHz}$ and the $30 \mathrm{GHz}$ were clamped iris structures. In addition the initial surface finish of the irises of both structures was quite poor. Both of these issues will be addressed when an HDS geometry [19] molybdenum structure will be tested in CTF3. Another question is whether the breakdown probability dependence is really exponential down to low values. This will require long and stable running periods.

A possible explanation for the origin of a breakdown rate and its functional dependence is fatigue due to local cyclic tensile stress. It has been proposed that the ultimate gradient is determined when the electrostatic pull caused by the local electric field, including the Fowler-Nordhiem derived field enhancement factor $\beta$, exceeds the material tensile strength limit [20,21]. In this model $\mathrm{rf}$ breakdown is initiated when a the pulling force of a local electric field site exceeds the tensile strength of the material 
and a clump of material is broken off. A logical extension of this argument is that the breakdown probability below the ultimate gradient is given by fatigue, the accumulation of defects, under the cyclic tension. The curves of fatigue as shown for example in [22] give qualitatively the correct functional form to fit the breakdown probability data. Fatigue data of molybdenum is planned for the fatigue test to determine if the same relationship holds as for the rf breakdown probability data.

\section{ACKNOWLEDGMENTS}

The author would like to sincerely thank Alberto Rodriguez, Alexej Grudiev, Chris Adolphsen, Igor Syrachev, Jim Norem, Mauro Taborelli, Raquel Fandos, Sami Tantawi, Steffen Döbert and Valery Dolgashev for numerous, lengthy and often animated discussions about the theory and measurement of breakdown - even when you don't agree with me!

\section{REFERENCES}

1. H. Braun, R. Corsini, A. de Roeck, A. Grudiev, S. Heikkinen, E.Jensen, M. Korostelev, D.Schulte, I. Syratchev, F.Tecker, W.Wuensch, F. Zimmermann, "Updated CLIC Parameters 2005", CLIC note 627.

2. Unpublished test in CTF2.

3. A. Grudiev, D. Schulte, W. Wuensch, "Optimum frequency and gradient for the CLIC main linac accelerating structure" Proceedings EPAC06.

4. W. Wuensch, C. Achard, S. Doebert, H.H. Braun, I. Syratchev, M. Taborelli, I. Wilson, "A Demonstration of High-Gradient Acceleration", Proceedings PAC2003 and CERN-AB-2003-048-RF.

5. W. Wuensch, "The Scaling of the Travelling-Wave Rf Breakdown Limit" CERN-AB-2006-013.

6. C. Adolphsen, "Advances in Normal Conducting Accelerator Technology From the X-Band Linear Collider Program", Proceedings PAC2005.

7. V. Dolgashev, "Effect of Rf Magnetic Fields and Input Power on Rf Breakdown Limit", Workshop on HighGradient RF, Argonne, October 2003.

8. W. Wuensch, A. Grudiev, S. Heikkinen, I. Syratchev, M. Taborelli, I. Wilson, S. Döbert, C. Adolphsen, "A High-Power Test of an X-Band Molybdenum-Iris Structure", Proceedings LINAC2004.

9. P. Wilson, these proceedings.

10. S. Döbert et. al., "High Gradient Performance of NLC/GLC X-Band Accelerating Structures", Proceedings PAC2005.

11. W. Wuensch et. al., "A High-Gradient Test of an $30 \mathrm{GHz}$ Molybdenum-Iris Structure”, Proceedings EPAC06.

12. R. Corsini et.al., "A High-Gradient Test of a $30 \mathrm{GHz}$ Copper Accelerating Structure", to be published in proceedings LINAC06.

13. W. Wuensch, C. Achard, H.H. Braun, G. Carron, R. Corsini, A. Grudiev, S. Tapio Heikkinen, D. Schulte, J. Sladen, I. Syratchev, F. Tecker, I. Wilson, "30 GHz Power Production in CTF3", Proceedings PAC2005.

14. J.W. Wang, G.A. Loew, R.J. Loewen, R.D. Ruth, A.E. Vlieks, I.Wilson, W.Wuensch, "SLAC/CERN High Gradient Tests of an X-band Accelerating Section", Proceedings PAC95 and CERN-SL-95-27 RF.

15. S. Döbert, "Gradient Limitations for High-Frequency Accelerators" Proceedings LINAC2004.

16. J.W. Wang et.al., "Progress Toward NLC/GLC Prototype Accelerator Structures", Proceedings LINAC2004.

17. V. Dolgashev, S. Tantawi, "RF Breakdown in X-Band Waveguides", Proceedings EPAC2002 and SLAC pub. 10355.

18. C. Adolphsen, "Normal Conducting RF Structure Test Facilities and Results", Proc. PAC2003 and SLAC-Pub9906.

19. A. Grudiev, W. Wuensch, "A newly designed and optimized CLIC main linac accelerating structure", Proceedings LINAC2004.

20. A. Grudiev, private communication.

21. J. Norem, these proceedings.

22. Sergio Calatroni, Samuli Heikkinen, Holger Neupert, Walter Wünsch, "Status of the Fatigue Studies on the CLIC Accelerating Structures", Proceedings EPAC06. 